\title{
Chronische Bandinstabilität des oberen Sprunggelenks
}

\author{
Hans Zwipp
}

\section{Zusammenfassung}

Die chronische Bandinstabilität des oberen Sprunggelenks (OSG) kann 3 funktionelle Einheiten betreffen. Am häufigsten kommt es nach Supinationstraumen mit fibularer Bandzerreißung und insuffizienter Verheilung des lateralen Bandkomplexes zum pathologischen Talusvorschub und varischer Taluskippung, d.h. zur chronischen anterolateralen Rotationsinstabilität (ALRI) des OSG $[2,5,11-13,17,23]$. Ähnlich häufig kann nach Pronationstraumen, meist nach unzureichend operierten Pronations-Eversions-(PE-) oder Pronations-Abduktions-Frakturen (PA-Frakturen) [3,8,9,23], seltener nach unerkannter oder bagatellisierter, rein ligamentärer Ruptur des vorderen und interossären Lig. tibiofibulare bzw. vermeintlich „isolierter Fraktur“ des Volkmann-Fragments eine chronische distale Syndesmoseninsuffizienz resultieren $[6,7,21,23,25]$. Die seltene Entität einer chronischen Bandinstabilität des medialen Bandkomplexes ist nach PE-IV-/PA-III-Fraktur, übersehenem Pronations-Eversions-Trauma (Stadium I) und/oder Ruptur/Partialruptur der Tibialis-posterior-Sehne $[10,24]$ beobachtbar, die i.d.R. mit einem Pes plano valgus vergesellschaftet ist. Für sog. Second-Stage-Rupturen [12] und die chronische ALRI-OSG werden heute statt Tenodesen [1,22] ausschließlich anatomische Rekonstruktionen der fehlverheilten fibularen Bänder empfohlen $[2,4,5,11-13,15,19,22,23]$. Zur operativen Behandlung der chronischen distalen Syndesmoseninsuffizienz wird im nordamerikanischen Raum die iatrogene Synostose zwischen Fibula und Tibia [15], im europäischen Raum die Ersatzplastik mit der Peronaeus-brevis-Sehne nach Castaing oder die anatomische 3-Punkt-Fixierung mit halber Peronaeus-longus-Sehne zum Ersatz aller 3 Bänder [8] empfohlen. Die seltene chronische Insuffizienz des Deltoids erfordert die verkürzende Re-Insertion meist in Addition mit einer varisierenden und/oder elongierenden Kalkaneus-Osteotomie [11].

\section{Chronic Ligament Instability of the Ankle Joint}

Chronic instability of the ankle joint may concern three entities: the fibular ligaments or distal syndesmotic ligaments and very rarely the deltoid ligament. The fibular ligament insufficiency results after failed fibular ligament healing after rupture due to a significant supination trauma producing anterior drawer and varus tilting of the talus defined as an anterolateral rotational instability (ALRI) of the ankle joint $[2,3,5,11-13,17,23]$. Distal syn- desmotic instability may result after PE fractures (PE: pronation-eversion), PA fractures (PA: pronation-abduction) due to lacking healing of the interosseous tibiofibular ligament $[3,8,9$, 23 ] as well as after pure ligament rupture especially after significant pronation trauma in hockey players $[6,7,21$, $23,25]$. Seldom is seen a chronic unstable deltoid after PE/PA fractures, PE trauma stage I and/or traumatic lesions of the posterior tibialis tendon $[11,24]$. For the lateral ankle ligament repair since the 1980s anatomic Broström-repair is recommended $[2,5,11,12,22]$, periostal flaps $[11,17,22,23]$ or the substitution with local or free autogenous tendons $[4,15,16,18,19,23]$. This to avoid functional limitation of supination, dorsiflexion and late ankle arthritis what is known from tenodesis procedures $[1,22]$. For chronic distal syndesmotic repair débridement and artificial synostosis between fibula and tibia is recommended [15], in Europe a Castaing-ligamentoplasty with the complete peroneus brevis or with half of peroneus longus tendon [8] to substitute all three syndesmotic ligaments anatomically. The rare indication for deltoid insufficiency needs anatomic re-inforcement of the deltoid often combined with hindfoot osteotomy to correct the pes planovalgus deformity $[11,24]$.

\section{Einleitung}

Die chronische Bandinstabilität des oberen Sprunggelenks (OSG) betrifft am häufigsten den fibularen Bandkomplex nach Ruptur mit inadäquater Behandlung und Fehlverheilung des rupturier-

OP-JOURNAL 2014; 30: 104-111

(c) Georg Thieme Verlag KG Stuttgart · New York DOI http://dx.doi.org/10.1055/s-0034-1383259 ten Lig. fibulotalare anterius (FTA) und des Lig. fibulocalcaneare (FC) im Rahmen eines Supinationstraumas [12,23], wobei Zwipp und Tscherne [22] zwischen einer „second-stage“-Ruptur, einem Riss in einer Ligamentnarbe, einer Reruptur (erneuter Riss in einem stabil verheilten fibularen Band) und einer chronischen Instabilität mit teils resorbierten, nicht mehr erkennbaren Bandstümpfen unterscheiden. Sie klassifizieren intraoperativ in narbig-stabil, narbig-instabil, dystop, elongiert und pseudarthrotisch fehlverheilte fibulare Bänder. Da FTA und FC in 74\% aller Fälle kombiniert zerreißen [23], kommt es i.d.R. bei insuffizienter Verheilung zur anterolateralen Rotationsinstabilität (ALRI) des OSG. Nach Pronations-Eversions-Traumen, meist im Rahmen einer PE-/PA-Fraktur des OSG oder einer rein ligamentären Syndesmosenruptur, kann es bei Nichterkennung mit insuffizienter Verheilung des Lig. tibiofibulare interosseum $\mathrm{zu}$ 

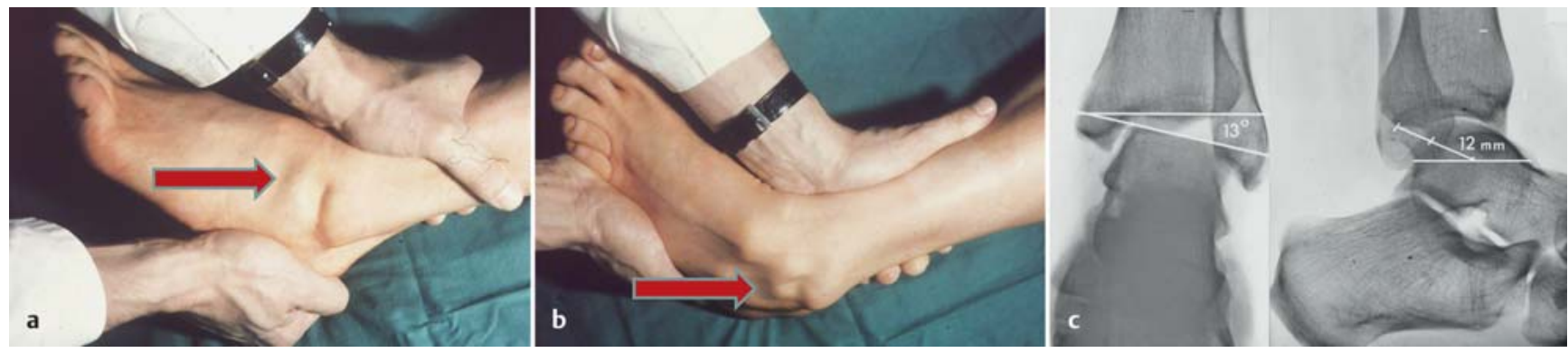

Abb. 1 a bis c Klinisch-radiologische Diagnostik der mechanischen Instabilität des lateralen OSG: a Testung der vorderen Schublade. b Varuskippung des Talus. Die Pfeile zeigen auf die Facies malleolaris lateralis tali, welche unter der Haut indirekt sichtbar wird. c Radiologische Stressuntersuchung zur Taluskippung und zum Talusvorschub mit standardisierter Ausmessung.

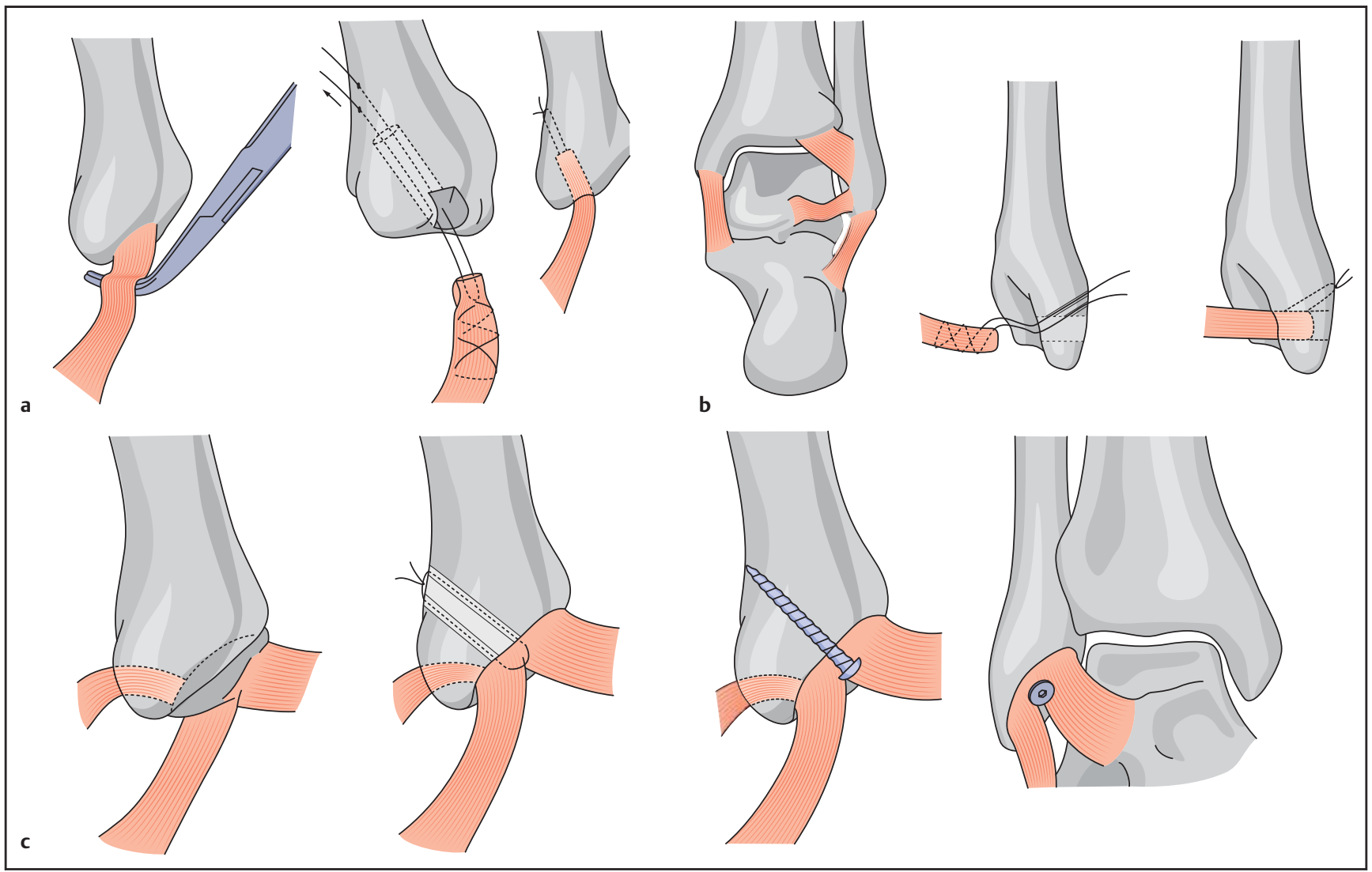

Abb. 2 a bis c Direkt anatomische Rekonstruktionstechniken bei insuffizienter Verheilung der vorhandenen fibularen Bandstrukturen: a Ablösung des elongierten Lig. fibulocalcaneare mit kleinem Knochenblock, um es unter Verkürzung in einen 4,5er-Bohrkanal am anatomischen Ort hineinzuziehen. b Verkürzende Re-Insertion eines elongierten Lig. fibulotalare posterius. c Technik der Re-Insertion eines pseudarthrotisch fehlverheilten Fragments mit anhängendem Lig. fibulotalare anterius und Lig. fibulocalcaneare nach Anfrischen der Pseudarthroseregion an der Fibula und mit Fixation des ossären Fragments mittels kleiner Zuggurtungs- oder Schraubenosteosynthese.

einer chronischen Instabilität des distalen Syndesmosenkomplexes kommen, was besonders bei professionellen Hockeyspielern beobachtbar ist $[6-8,21]$. Extrem selten hingegen kann eine chronische Instabilität des Lig. deltoideum nach PE-/PA-Fraktur oder einer Pronations-Eversions-Verletzung (Typ I) gesehen werden, was arthroskopisch häufiger feststellbar ist als allgemein erwartet [11].

\section{Chronische anterolaterale Rotations- instabilität des oberen Sprunggelenks (ALRI-OSG)}

\section{Anamnese und Befund}

Eine funktionelle Instabilität des OSG kann gegenüber einer mechanischen Instabilität nur radiologisch mittels Stressaufnahme für Taluskippung und -vorschub abgegrenzt werden.
Gangunsicherheit auf unebenem Gelände nach einem vorausgegangenen relevanten Supinationstrauma des Fußes, eine nur bedingte Sportfähigkeit (notwendige Knöchelbandage) sowie die Angst vor dem rezidivierenden Umknicken des Fußes in Sinne der Supination sind typische Hinweise für eine chronische Instabilität des OSG, welche den Ausschluss einer rein funktionellen Instabilität erfordern. Typische Befunde einer mechanischen Instabilität finden sich bei der klinischen Untersuchung im Sinne des Nachweises 


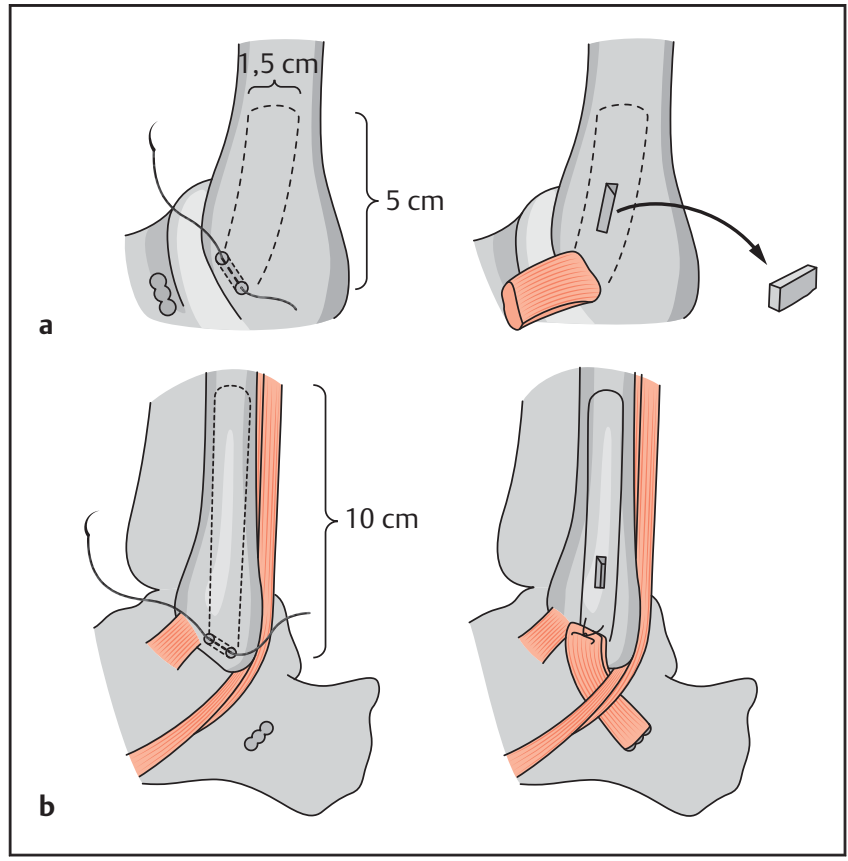

Abb. $3 \mathbf{a}$ und $\mathbf{b}$

Periostlappenersatzplastik bei atrophem oder nicht mehr rekonstruierbarem FTA oder FC: a Zum Ersatz des FTA wird ein gedoppelter, mind. $5 \mathrm{~cm}$ langer und $1,5 \mathrm{~cm}$ breiter Periostlappen präpariert und nach Doppelung an der Fibula mit transossärer Naht gesichert und anschließend am gelenknahen Talushals mit kleinem freiem Knochenblock eingedübelt, heute mit bioresorbierbarem „bone anchor“ fixiert. b Analoges Verfahren zum Ersatz des FC, wobei hier zur notwendigen Doppelung ein Perioststreifen von mind. $10 \mathrm{~cm}$ Länge präpariert werden muss. einer vorderen Schublade und einer Varuskippung des Talus im Sprunggelenk (Abb. 1 a,b). Beweisend hierfür sind ein pathologischer Talusvorschub kombiniert mit einer pathologischen varischen Kippung des Talus anhand gehaltener Röntgenstressaufnahmen (Abb. 1 c), wodurch eine rein funktionelle Instabilität sicher ausgeschlossen werden kann. Bei jahrelang bestehender ALRI-OSG können zusätzlich relevante Knorpelläsionen in $5 \%$, eine Tendovaginitis der Peronäalsehnen in $14 \%$, eine Synovitis des OSG in 24\% und radiologische Arthrosezeichen im Sinne Bargon I bis II in 38\% gesehen werden [22].

\section{Therapie}

Konservative Behandlung ALRI-OSG

- Eigenreflex- und Pronatorentraining

- lateraler Schuhabsatzkeil von $0,5 \mathrm{~cm}$

- Rückfußpronierende Schuheinlagen

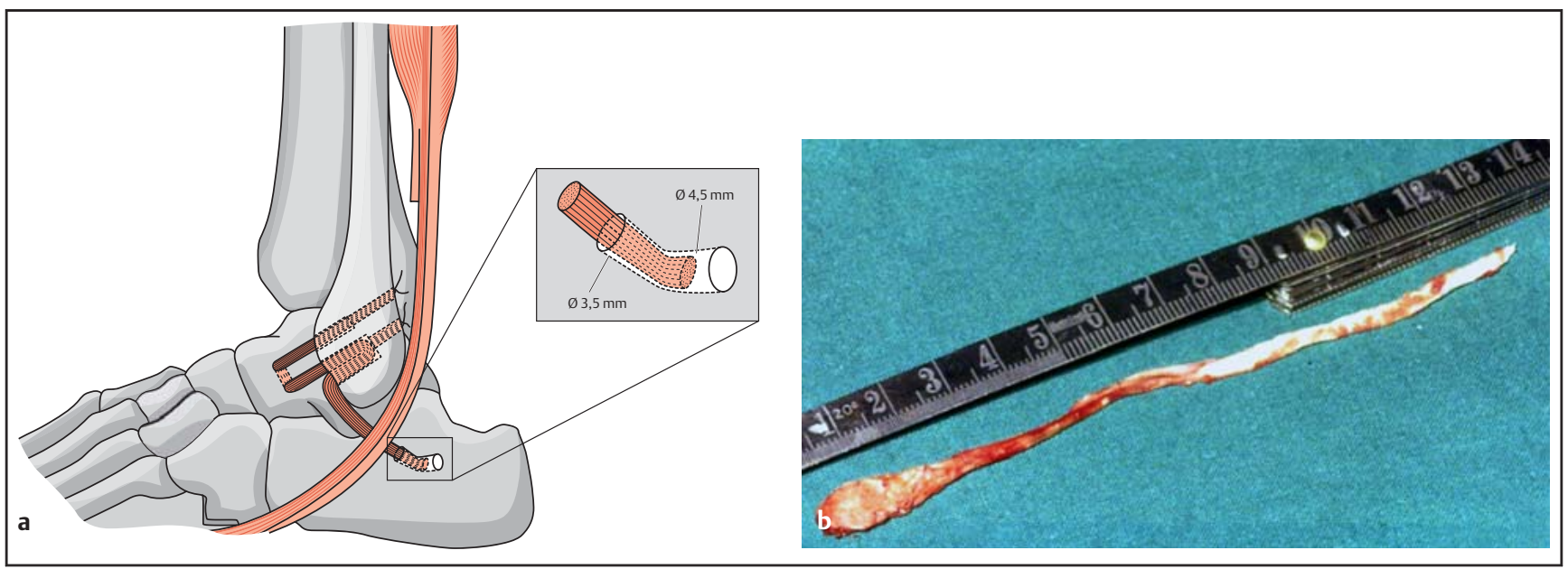

Abb. 4 Bei atrophen Verhältnissen des FTA und FC wird im eigenen Vorgehen ein freier halber Peronaeus-brevis-Span mit kleinem Knochenblock von der Basis des 5. Mittelfußknochens zum anatomischen Ersatz beider Bänder verwendet. Die unterschiedlich starken Bohrungen am Kalkaneus ermöglichen eine gute Verankerung durch den in den Kalkaneus hineingezogenen Knochenblock.
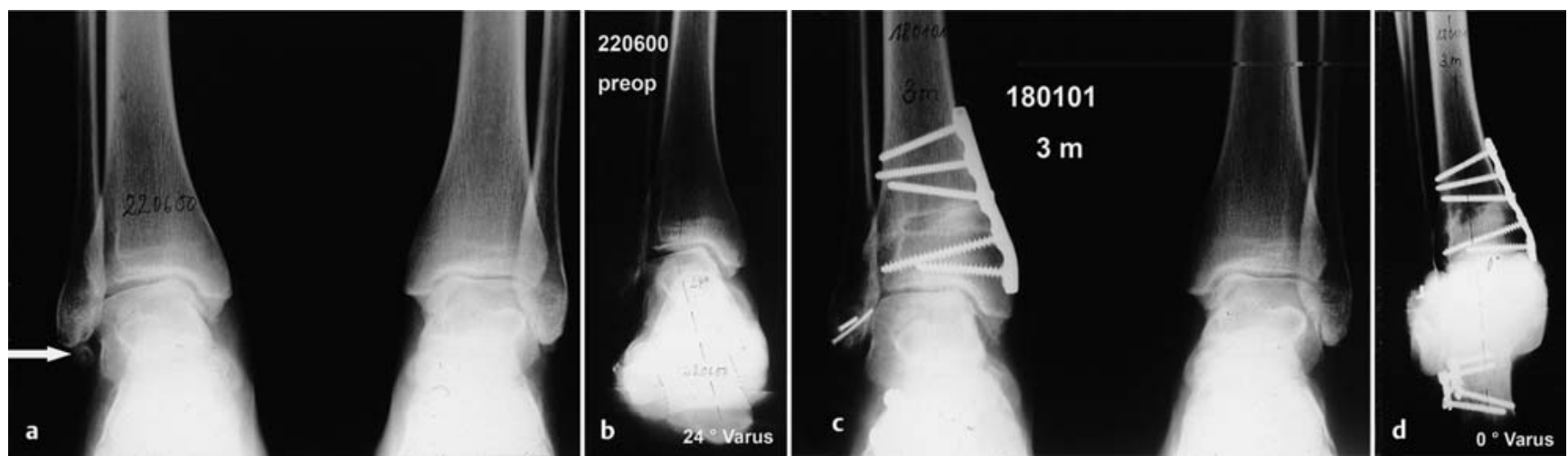

Abb. $\mathbf{5}$ a bis d Doppelosteotomie-Technik bei relevantem Rückfußvarus und chronischer ALRI. Hier wird bei pseudarthrotischer Fehlverheilung des FTA und FC (a) und $24^{\circ}$ Rückfußvarus in der Saltzman-Projektion (b) im eigenen Vorgehen neben der Fixation des pseudarthrotischen Fragments an der Fibula mit Minischrauben eine valgisierende Kalkaneus-Osteotomie nach Dwyer durchgeführt. Der entnommene Kalkaneuskeil wird zur zusätzlichen Valgisierung nach supramalleolärer Tibiaosteotomie interponiert und mit 3,5er-Platte gesichert (c), um durch diese Doppelosteotomie die Rückfußachse zu korrigieren (d). 

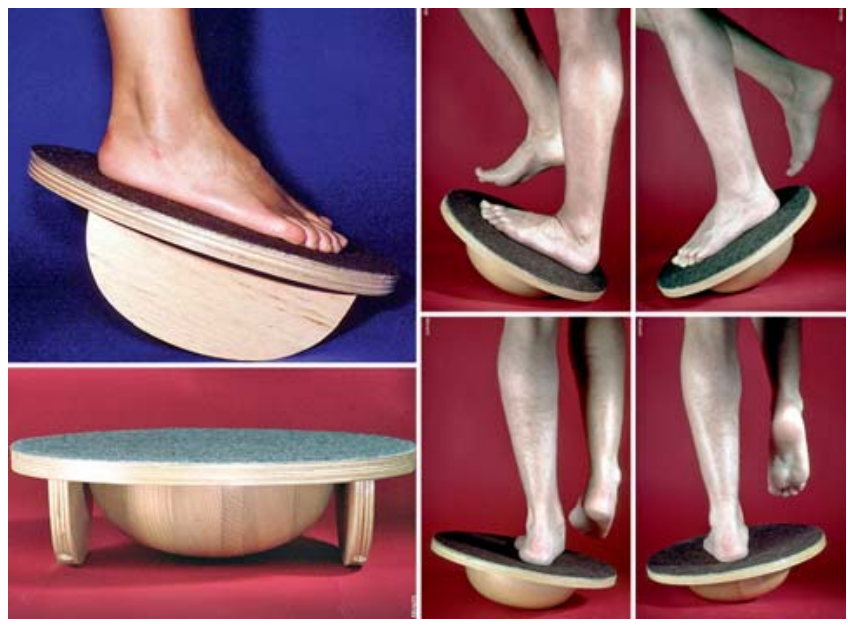

Abb. 6 Die Nachbehandlung nach fibularen Bandrekonstruktionen beinhaltet nach 6-wöchiger Protektion in einer supinatorisch hemmenden Orthese (z. B. Caligaloc ${ }^{\circledR}$ ) grundsätzlich das Eigenreflextraining auf dem Therapiekreisel. Dies sollte initial im Sinne der eindimensionalen Schiffschaukel, anschließend mehrdimensional mit Halbkugel beidbeinig, erst danach einbeinig mind. in 10 Therapieeinheiten durchgeführt werden.

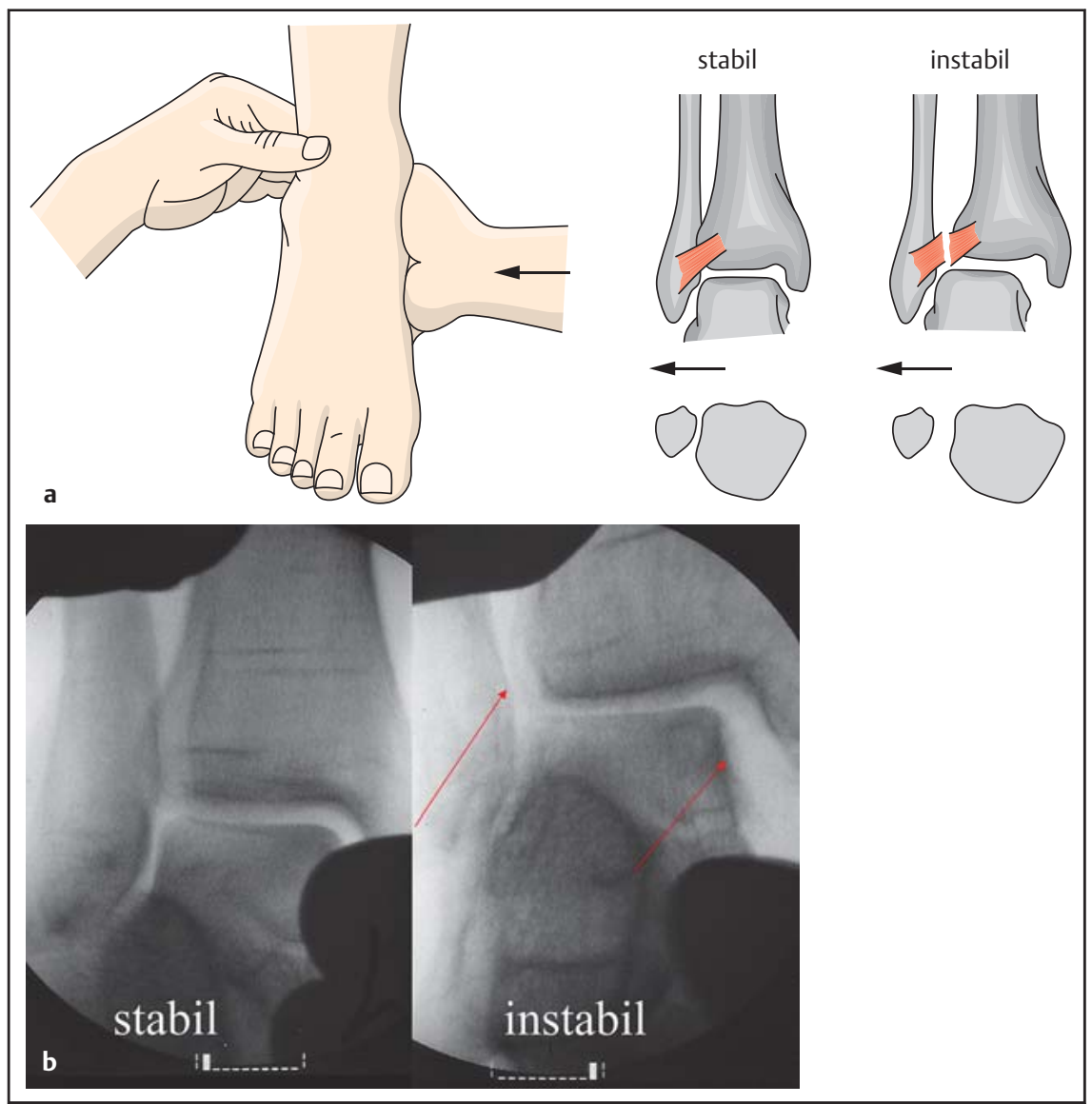

Abb. 7a und $\mathbf{b}$ Klinisch-radiologische Stresstestung der Sprunggelenksgabel bei Verdacht auf Ruptur des Lig. tibiofibulare interosseum: a Unter Aussparung der Fibula, z. B. mit der linken Hand, wird mit der rechten Hand der Kalkaneus nach lateral gedrückt, wobei klinisch ggf. eine leicht federnde Instabilität gespürt werden kann. Zur sicheren Differenzialdiagnostik eines Risses nur des vorderen Syndesmosenbands gegenüber einer zusätzlichen Ruptur des Lig. tibiofibulare interosseum wird entsprechend der klinischen Testung das OSG durchleuchtet (b). Dadurch kann im Seitenvergleich ein erweiterter „espace clair“ und ein pathologisch erweiterter „medial clear space“ bei Ruptur des Lig. tibiofibulare interosseum dokumentiert werden.

Vor der Entscheidung zu einer operativen Maßnahme sollte grundsätzlich im 1. Schritt der Behandlung nach Diagnosestellung einer mechanischen OSG-Instabilität des fibularen Bandapparats eine konsequente, konservative Therapie mit Eigenreflex- und Pronatorentraining mit wenigstens 10 Therapieeinheiten erfolgen. Kommt es darunter zu einer dynamischen Stabilisierung des OSG, kann eine operative Maßnahme zurückgestellt werden. Bei Versagen konservativer Maßnahmen, einschließlich Schuhaußenranderhöhung um $0,5 \mathrm{~cm}$ oder pronatorisch wirkender Schuheinlage, kommen bei fehlenden Kontraindikationen zur Operation anatomische Rekonstruktionstechniken in Betracht.

\section{OP-Techniken}

Je nach intraoperativem Befund und zusätzlicher Pathologie (z.B. Rückfußvarus) kommt in erster Linie die direkte Bandrekonstruktion eines ossär-pseudarthrotisch, dystop oder elongiert fehlverheilten Bandes (Abb.2) in Betracht, bei narbig instabil verheiltem FTA oder FC die gedoppelte Periostlappenplastik (Abb. 3). Nur bei notwendigem Ersatz beider Bänder ist im eigenen Vorgehen die anatomische Ersatzplastik mit halber Peronaeus-brevis-Sehne mit Knochenblock von der Basis des Metatarsale V empfehlenswert (Abb.4). Bei chronischer ALRI-OSG kombiniert mit Rückfußvarus hat sich die valgisierende Kalkaneus- und/oder zusätzliche valgisierende supramalleoläre Osteotomie bewährt (Abb. 5).

\section{Nachbehandlung}

Statt Tenodese immer anatomische Rekonstruktion, Periostlappenplastik oder anatomische Sehnenersatzplastik. Bei Rückfußvarus zusätzliche Valgisierungsoperation des Fersenbeins.

Bei stabiler anatomischer Rekonstruktion kann nach Wundheilung in einer Knöchelorthese (z.B. Caligaloc ${ }^{\circledR}$ ) und unter Vollbelastung des Fußes nachbehandelt werden. Danach sollte grundsätzlich ein Eigenreflex- und Pronatorentraining erfolgen (Abb. 6). Ratsam ist die anschließende Nachbehandlung mit Verordnung einer lateralen Absatzerhöhung um $0,5 \mathrm{~cm}$ oder einer pronatorisch wirkenden Einlage für einige Monate postoperativ.

\section{Chronische distale Syndesmoseninstabilität}

\section{Anamnese und Befund}

Pronationstraumen bei Hockeyspielern führen oft zur vollständigen Zerreißung des distalen Syndesmosenkomplexes.

Die Patienten berichten i.d. R. über eine zuvor erlittene Knöchelfraktur im Sinne einer PE- oder PA-Fraktur des OSG, die 

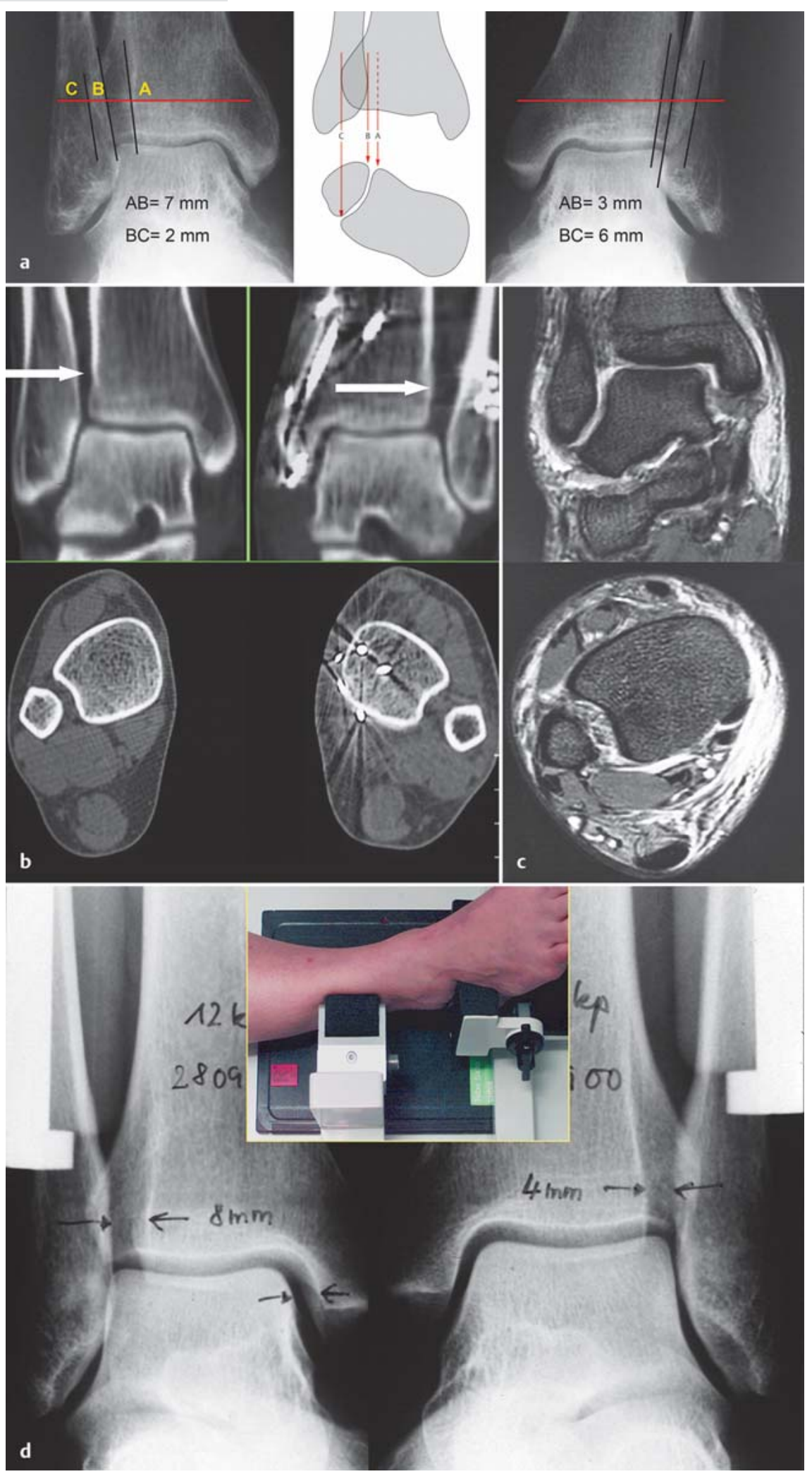

Abb. $\mathbf{8}$ a bis d Radiologischer Nachweis einer chronischen Syndesmoseninstabilität: a Bei Belastungsaufnahmen im Stehen mit $20^{\circ}$ Innenrotation beider Füße sollte der „espace clair“ unter $5 \mathrm{~mm}$ betragen und das „overlapping“ mehr als $5 \mathrm{~mm}$. Der „medial clear space“ sollte nicht mehr als $2,5 \mathrm{~mm}$ betragen. $\mathbf{b}$ Die CT zeigt in der frontalen Schicht (oben) den pathologisch erweiterten „espace clair“. In der koronaren Schichtung (unten) ist die erweiterte Distanz zwischen Fibula und Tibia prima vista erkennbar. c Die MRT kann sowohl in der frontalen als auch in der koronaren Schicht die instabile Vernarbung des Lig. tibiofibulare interosseum deutlich machen. $\mathbf{d}$ Standardisierte Syndesmosenstressaufnahmen mit dem Telos-Gerät zeigen rechts neben dem erweiterten „espace clair“ den erweiterten "medial clear space“. operativ versorgt worden war. Seltener wird über eine konservativ behandelte, vermeintlich „isolierte Volkmann-Fraktur" oder ein relevantes Pronationstrauma z.B. beim Hockeysport [7,8,22] berichtet. Typische Beschwerden sind die Gangunsicherheit, besonders beim Treppabsteigen, das Instabilitätsgefühl in der Knöchelgabel meist beim Gehen auf unebenem Gelände sowie der häufig angegebene Schmerz im Bereich des anteromedialen OSG. Dieser ist erklärbar durch die pathologische Führung des Talus aufgrund der nach dorsal abweichenden Fibula, was zum Impingement zwischen medialem Talus und Innenknöchel führt. Sowohl bei frischer als auch bei veralteter oder chronischer Syndesmoseninstabilität ist der klinische Stabilitätstest (Abb.7a) wegweisend und die Durchleuchtungsstressaufnahme (Abb. 7b) beweisend.

Ein Beleg für die rein ligamentäre distale Syndesmoseninsuffizienz sind entweder der pathologisch erweiterte „espace clair“" (>5 mm), das verminderte „overlapping“ $(<5 \mathrm{~mm})$ bzw. der pathologisch erweiterte „medial clear space“ (> 2,5 mm) anhand von OSG-Belastungsaufnahmen im Stehen mit $20^{\circ}$ Innenrotation beider Füße (Abb. 8a). Alternative Beweise sind die Erweiterung der Knöchelgabel in der axialen und/oder frontalen CT-/MRTSchnittebene (Abb. 8b und c) bzw. die standardisiert gehaltene Syndesmosenröntgenstressaufnahme (Abb. 8d).

\section{Therapie}

Da eine konservative Behandlung nach vollständiger Diagnostik mit Ausschluss einer Fibulaverkürzung oder -fehlrotation mit dem Nachweis einer rein ligamentären distalen Syndesmoseninsuffizienz nicht zielführend ist und nur bei Kontraindikation zur Operation als Orthesenbehandlung in Betracht käme, ist i.d.R. eine operative Korrektur angezeigt. Da im eigenen Verständnis eine artifizielle Synostose zwischen Fibula und Tibia seit den Untersuchungen von B.G. Weber [20] als präarthrotische Deformität gilt, wird im europäischen Raum die Ligamentersatzplastik empfohlen. Während Castaing (zitiert in [23]) mit einer ganzen Peronaeus-brevis-Sehne das vordere und hintere Syndesmosenband ersetzt, wird im eigenen Vorgehen [8] mit der distal gestielten halben Peronaeus-longus-Sehne das vordere interossäre und das hintere distale Syndesmosenband im Sinne einer 3-Punkt-Fixierung anatomisch ersetzt (Abb.9). Für 


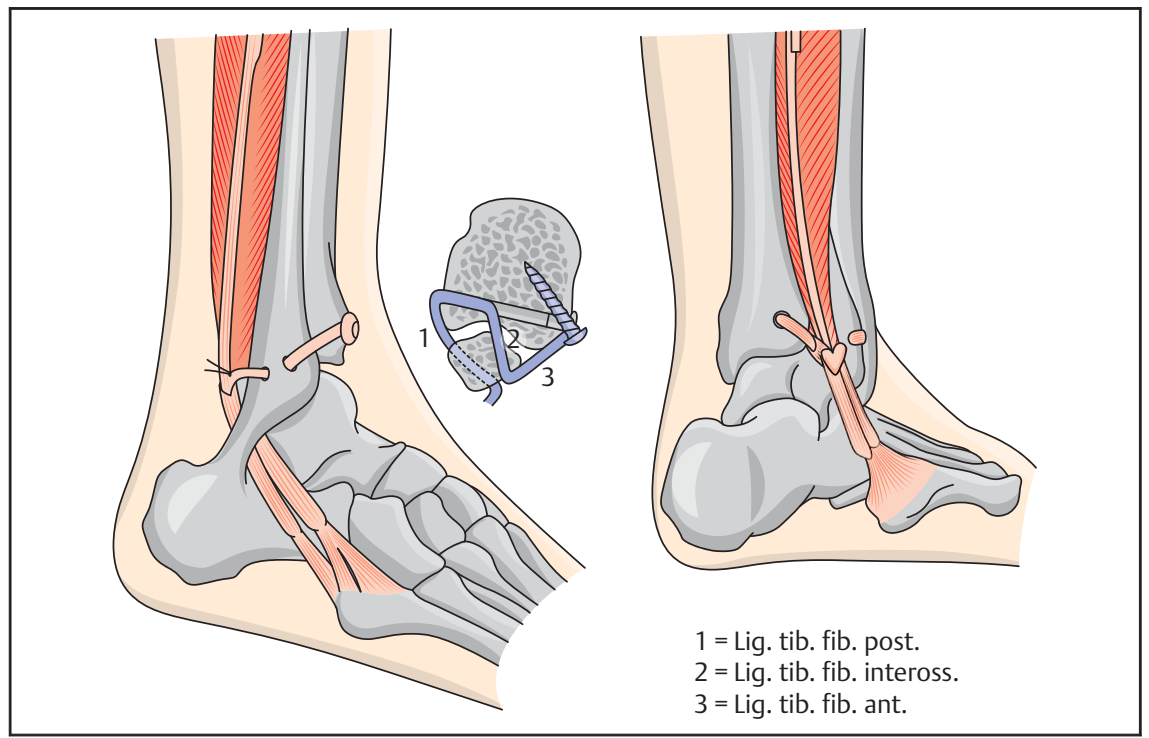

Abb.9 Anatomische Ersatzplastik des distalen Syndesmosenkomplexes mit 3-Punkt-Fixierung des vorderen, mittleren und hinteren Zügels der distal gestielten Peronaeus-longus-Sehne.

die sichere Einheilung des transponierten Sehnenspans wird eine Syndesmosenschraube gesetzt [8], die nach 8 Wochen in Lokalanästhesie entfernt werden kann. Bei zusätzlich notwendiger Osteotomie der Fibula zur Verlängerung, Rotationskorrektur oder zwecks Exploration einer Volkmann-Fragment-Verheilung in ca. $10 \%$ der Fälle, wird in der persönlichen Technik die halbe Peronaeus-brevis-Sehne zum Ersatz nur des mittleren und vorderen Bandes verwendet (Abb. 10).

Die anatomische Syndesmosenersatzplastik aller 3 Syndesmosenbänder ist physiologischer als eine artifiziell angestrebte Synostose.

\section{Nachbehandlung}

Nach Wundheilung erfolgt diese grundsätzlich im Unterschenkelgehcast in Rechtwinkelstellung des Fußes mit 20 kp Teilbelastung für 8 Wochen. Nach entsprechender Weichteilbehandlung wird nach der 8. Woche postoperativ die Syndesmosenstellschraube in Lokalanästhesie entfernt und eine krankengymnastische Übungsbehandlung mit zunehmender Vollbelastung in den folgenden 3-4 Wochen angeordnet.

\section{Chronische Bandinstabilität des Lig. deltoideum}

Nach Empfehlungen von Hintermann [10] wird das elongierte Deltoid unter Verkürzung re-inseriert. Bei relevantem Rückfußvalgus wird eine additive vari- sierende und/oder verlängernde Kalkaneusosteotomie zusätzlich durchgeführt.

\section{Diskussion}

Während bei der chronischen ALRI-OSG bis in die 1980er-Jahre hinein über 50 verschiedene Operationsmethoden empfohlen wurden, haben sich diese seit den Erkenntnissen zur supinationshemmenden Wirkung der Tenodesen kombiniert mit posttraumatischer Arthrose im Langzeitergebnis auf heute wenige, aber rein anatomisch orientierte Operationstechniken mit autologem Gewebe reduziert [4,11-13,15-19,22,23]. Wenngleich Broström bereits 1966 [2] auf einfache, an der Anatomie und Biomechanik orientierte Techniken hinwies, haben sich anatomische Rekonstruktionsverfahren erst seit den 1980er-Jahren etabliert, obwohl noch 1994 an deutschen Kliniken in 33\% der Fälle einer chronischen ALRI-OSG $(\mathrm{n}=4845)$ Tenodesetechniken angewandt wurden [1]. Während im eigenen operativen Vorgehen bei isolierter OSG- oder kombinierter OSG-/USG-Instabilität bis in die 1990erJahre noch die sog. Elmsilie-Tenodese durchgeführt und empfohlen wurde [23], ist diese in den letzten 10 Jahren im eigenen Vorgehen nicht mehr notwendig geworden. Dies deshalb, weil durch die transossär geführte Verkürzung eines elongierten Lig. fibulocalcaneare immer eine ausreichende Stabilität des subtalaren Gelenks erzielt werden konnte. In der Literatur werden für anatomisch-rekonstruktive Verfahren wie die direkte Bandrekonstruktion, die
Periostlappenplastik oder der Ersatz von FTA und FC mit gestielten oder freien Sehnenspänen sehr gute und gute Resultate sowohl in Bezug auf die Funktion als auch auf die Stabilität beschrieben [4, 11-13,15-19,22,23]. Eine kürzliche anatomische Studie [3] zeigt die unmittelbare nahe Nachbarschaft von FTA und FC sowie die häufige Doppelanlage des FTA, sodass die anatomisch-orientierten Ersatzplastiken $[4,15,16,18,19$, 23] Grundlagen für die volle Funktion des Fußes bei biomechanischer Stabilität darstellen.

Während in der nordamerikanischen Literatur $[9,15]$ bei chronischer distaler Syndesmoseninsuffizienz die artifizielle Synostose zwischen Fibula und Tibia empfohlen wird, sind im europäischen Raum Syndesmosenersatzplastiken als physiologische Methoden empfohlen [8, 23]. Im eigenen Vorgehen konnten durch Verwendung der halben Peronaeus-longus-Sehne in weit über 100 Fällen keine Nachteile der Sehnenfunktion gesehen werden. Die eigenen Ergebnisse zeigen auch im Langzeitverlauf, dass nach Ersatz des distalen Syndesmosenbands trotz bereits nachweisbarer arthrotischer Veränderungen nach PE-/PA-Frakturen nicht nur bei Rekonstruktion aller 3 Syndesmosenbänder, sondern auch bei einfacher anatomischer Ersatzplastik nur des vorderen und interossären Syndesmosenbands die bestehenden $\mathrm{Ar}$ throsezeichen i.d.R. nicht progredient sind, sondern Stabilität und Beschwerdefreiheit für den Patienten bedingen (Abb. 10).

Aufgrund der geringen Berichte in der Literatur [10] und der eigenen Erfahrung in nur 3 Fällen einer anatomischen Korrektur des insuffizienten Lig. deltoideum kann die Effizienz der beschriebenen Methodik nicht ausreichend beurteilt werden. Wesentlich erscheinen vielmehr additive Maßnahmen zur Beseitigung des Rückfußvalgus und der pathologischen Innenrotation des Talus durch die varisierende und/oder elongierende Kalkaneusosteotomie.

\section{Fazit für die Praxis}

Nach der heutigen internationalen Literatur werden bei chronischer anterolateraler Rotationsinstabilität des OSG Tenodesetechniken aufgrund ihrer supinationshemmenden Wirkung des Fußes mit Begünstigung von Spätarthrosen des OSG abgelehnt. Stattdessen werden direkt anatomische Rekonstruktionen 


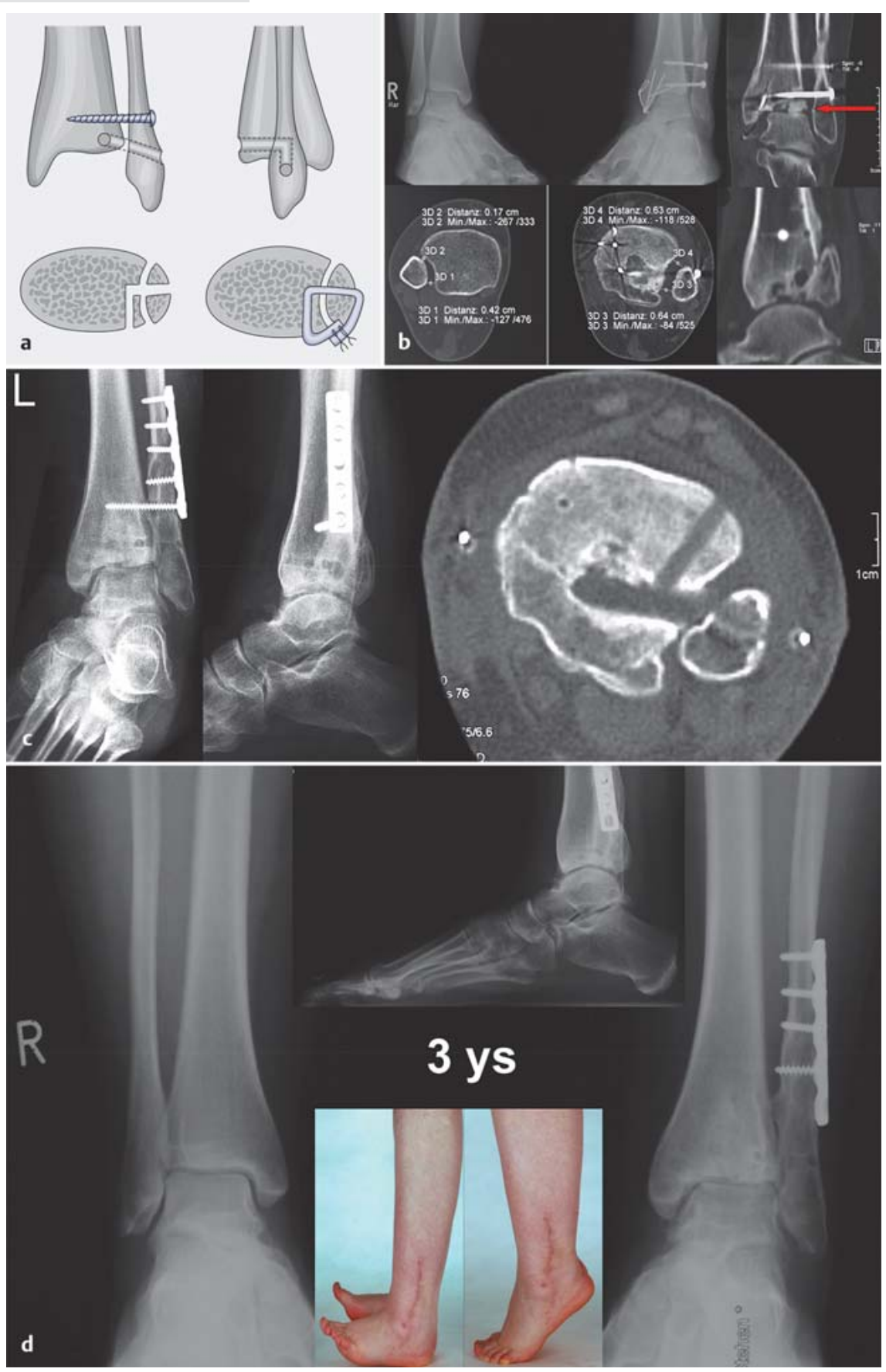

Abb. 10a bis d Bei chronischer distaler Syndesmoseninsuffizienz und zusätzlich notwendiger Osteotomie der Fibula bei Verkürzung, Fehlrotation, Pseudarthrose oder notwendiger Osteotomie zur Korrektur eines fehlverheilten Volkmann-Fragments empfiehlt sich die einfachere Variante der distalen Syndesmosenersatzplastik mit freiem halbem Peronaeus-brevis-Span (a) zum Ersatz des Lig. tibiofibulare interosseum und des Lig. tibiofibulare anterius. b Fallbeispiel einer Fehlverheilung des Volkmann-Fragments mit partieller Nekrose des Tubercule de Chaput kombiniert mit Erweiterung der Incisura fibularis tibiae 9 Monate nach auswärtiger Versorgung einer PE-Fraktur bei einer 29-jährigen Patientin. Nach Osteotomie der Fibula zur Überprüfung der evtl. notwendigen Osteotomie des fehlverheilten Volkmann-Fragments wird die Stabilität der Sprunggelenksgabel mit halbem freiem Peronaeus-brevis-Span - wie in a schematisch gezeigt - wiederhergestellt. c Das intraoperative Röntgenbild (links) zeigt die Osteosynthese der Fibula mit Stellschraube durch das distale Schraubenloch und einen kaum erkennbaren Gelenkspalt. Die postoperative CT (rechts) lässt die Bohrkanäle und Position der Fibula erkennen. d Die 3-Jahres-Kontrolle zeigt hingegen eine wesentlich bessere Gelenksituation. Die Patientin ist völlig beschwerdefrei und hat eine sehr gute Funktion des OSG. der fibularen Bänder, Periostlappenersatzplastiken und der gestielte, freie, autologe Ersatz beider Bänder mit Sehnengewebe empfohlen. Zur Behandlung der chronischen distalen Syndesmoseninsuffizienz wird im nordamerikanischen Raum die tibiofibulare Arthrodese, im europäischen Raum die anatomische Ersatzplastik, möglichst aller 3 tibiofibularen Bänder, mit autologem Sehnengewebe empfohlen. Die seltene Deltoidinsuffizienz benötigt neben direkter anatomischer Rekonstruktion meist die additive varisierende und/oder elongierende Kalkaneusosteotomie. Zur Vermeidung der 3 chronischen Entitäten werden die Erkennung einer akuten Instabilität durch exakte klinisch-radiologische Stabilitätsprüfung gefordert sowie die Sicherstellung der Ligamentheilung. Diese erfolgt bei akuter ALRI mittels supinationshemmender Orthese (z.B. Caligaloc $\left.{ }^{\circledR}\right)$, bei isolierter Ruptur des Lig. fibulotalare anterius die funktionelle Orthesenbehandlung ohne einen pronierenden Rückfußkeil der CalicalocSchiene (d.h. Abschleifen desselben) oder die Verwendung anderer nicht pronierender Orthesen. Bei akuter Ruptur des Lig. tibiofibulare interosseum hilft aufgrund der relevanten Instabilität nur eine operative Revision der Syndesmose mit korrekter Positionierung der Fibula in die Incisura tibiae fibularis und anschließender Fixation mittels zweier Stellschrauben, was möglichst intraoperativ mit 3-D-CT oder postoperativ mittels seitenvergleichendem CT überprüft werden soll, um die chronische Syndesmoseninsuffizienz zu vermeiden.

\section{Literatur}

${ }^{1}$ Becker HP, Zeithammel G, Danz B et al. Klinische und röntgenologische 5-Jahres-Ergebnisse nach modifizierter Evans-Plastik bei chronischer Außenbandinstabilität des Sprunggelenks. Unfallchirurg 1999; 98: 333337

2 Broström L. Sprained ankles. IV. surgical treatment of "chronic" ligament ruptures. Acta Chir Scand 1966; 132: 551

3 Boszotta H, Sauer G. Die chronische fibulare Bandinsuffizienz am oberen Sprunggelenk. Spätergebnisse nach modifizierter WatsonJones-Plastik. Unfallchirurg 1989; 92: 11-16

4 Clanton TO, Campbell KJ, Wilson KJ et al. Qualitative and quantitative anatomic investigation of the lateral ligaments for surgical reconstruction procedures. J Bone Joint Surg Am 2014; 96: e98

5 Colville MR, Gronde RJ. anatomic reconstruction of the lateral ankle ligaments using a split peroneus brevis tendon graft. Am J Sports Med 1995; 23: 210-213

${ }^{6}$ Czaja S, Müller W. Die anatomische Rekonstruktion des fibulotarsalen Bandkomplexes bei chronischer Bandinstabilität am Sprunggelenk. Operat Orthop Traumatol 1998; 10: 253-264 
${ }^{7}$ Flik K, Lyman S, Marx RG. American collegiate men's ice hockey: an analysis of injuries. Am J Sports Med 2005; 33: 183-187

8 Gerber JP, Williams GN, Scoville CR et al. Persistant disability associated with ankle sprains: a prospective examination of an athletic population. Foot Ankle Int 1998; 19: 653-660

${ }^{9}$ Grass R, Rammelt S, Biewener A et al. Peroneus longus ligamentoplasty for chronic instability of the distal tibiofibular syndesmosis. Foot Ankle Int 2003; 24: 392-397

${ }^{10}$ Hansen ST. Functional Reconstruction of the Foot and Ankle. Repair of distal tibiofibular Syndesmosis. Philadelphia: Williams \& Wilkins; 2000: 507-508

11 Hintermann B, Valderrabano $V$, Boss $A$ et al. Medial ankle instability: an exploratory, prospective study of fifty-two cases. Am J Sports Med 2004; 32: 183-190

12 Karlsson J, Bergsten T, Lansinger O et al. Surgical treatment of chronic lateral instability of the ankle joint. A new procedure. Am J Sports Med 1989; 17: 268-273

13 Knop C, Knop C, Thermann $H$ et al. Die Behandlung des Rezidivs einer fibularen Bandruptur. Ergebnisse einer prospektiv-randomisierten Studie. Unfallchirurg 1999; 102 : 23-28

${ }^{14}$ Krips $R$, van Dijk CN, Lehtonen $H$ et al. Sports activity level after surgical treatment for chronic anterolateral ankle instability. A multicenter study. Am J Sports Med 2002; 30: 13-19

15 Olson K, Dairyko GH jr., Toolan BC. Salvage of chronic instability of the syndesmosis with distal tibiofibular arthrodesis: functional and radiographic results. J Bone Joint Surg Am 2011; 93: 66-72

16 Paterson $R$, Cohen B, Taylor D et al. Reconstruction of the lateral ligaments of the ankle using semi-tendinosus graft. Foot Ankle Int 2000; $21: 413-419$

17 Richter J, Volz R, Immendörfer M et al. Rekonstruktion des Außenbandapparats mittels Kniebeugesehnentransplantat bei chronischer Außenbandinstabilität des OSG. Oper Orthop Traumatol 2012; 24: 50-60

18 Rudert M, Wülker $N$, Wirth CJ. Reconstruction of the lateral ligaments of the ankle using a regional periosteal flap. J Bone Joint Surg Br 1997; 79: 446-451

${ }^{19}$ Sugimoto K, Takakura Y, Tsukasa $K$ et al. Reconstruction of the lateral ankle ligaments with bone-patellar tendon-graft in patients with chronic ankle instability: a preliminary report. Am J Sports Med 2002; 30: 340-346

20 Takao M, Oae K, Uchio Y et al. Anatomical reconstruction of the lateral ligaments of the ankle with a gracilis autograft: a new technique using an interference fit anchoring system. Am J Sports Med 2005; 33: 814-823

${ }^{21}$ Weber BG. Die Verletzungen des oberen Sprunggelenkes. 2. Aufl. Bern, Stuttgart, Wien: Hans Huber; 1972
22 Wright RW, Barile RJ, Surprenant DA et al. Ankle syndesmosis sprains in national hockey league players. Am J Sports Med 2004; 32: 1941-1945

${ }^{23}$ Zwipp H, Tscherne H. Zur Behandlung der chronischen antero-lateralen Instabilität des oberen Sprunggelenkes: direkte Bandrekonstruktion - Periostlappenplastik - Tenodese. Unfallheilkunde 1984; 87: 405-415

${ }^{24}$ Zwipp H. Chirurgie des Fußes. Wien, New York: Springer; 1994

${ }^{25}$ Zwipp H, Dahlen C, Amlang M et al. Verletzungen der Sehne des M. tibialis posterior. Orthopäde 2000; 29: 251-259

${ }^{26}$ Zwipp H, Rammelt S. Neue Entwicklungen zur Diagnostik und Therapie bei OSG-Distorsionen und -Bandrupturen. Passion Chirurgie (BDC) 2012; 2: Artikel 03-0

Prof. Dr. med. Hans Zwipp

Ärztlicher Direktor

UniversitätsCentrum für

Orthopädie und Unfallchirurgie

Universitätsklinikum Dresden

Fetscherstraße 74

01307 Dresden

hans.zwipp@uniklinikum-dresden.de 